\title{
An Extremely Rare Isolated Hyoid Bone Fracture in a Patient Involved in a Traffic Accident
}

\author{
Mustafa Ozturk ${ }^{\mathrm{a}}$, Ozgur Sogut ${ }^{\mathrm{a}, \mathrm{b}}$, Mehmet Yigit ${ }^{\mathrm{a}}$, Onur Kaplan ${ }^{\mathrm{a}}$, \\ Ozlem Tataroglu ${ }^{\mathrm{a}}$, Demet Tas ${ }^{\mathrm{a}}$
}

\begin{abstract}
Hyoid fractures in victims of strangulation and hanging are well defined. Nevertheless, an isolated hyoid bone fracture caused by blunt neck trauma is extremely rare and accounts for only $0.002 \%$ of all fractures. The diagnosis of a patient with a hyoid bone fracture can be difficult. This type of fracture may go unseen during a physical examination, causing a life-threatening airway obstruction. We present a case of a young male with an isolated fracture of the hyoid bone induced by direct blunt neck trauma. The patient complained of anterior neck pain. A physical examination revealed tenderness in the neck, but no respiratory distress or limited range of motion of the neck was detected. A cervical computed tomography scan revealed a bony fracture involving the right greater horn of the hyoid bone with adjacent soft tissue swelling. A hyoid bone fracture should be considered when neck tenderness and pain are detected following blunt trauma to the neck region.
\end{abstract}

Keywords: Blunt trauma; Cervical computed tomography; Conservative management; Hyoid bone fracture

\section{Introduction}

Most hyoid bone injuries are due to strangulation and hanging [1]. Hyoid bone fractures are quite rare, including in pediatric patients [2]. The incidence of hyoid bone fractures in patients with trauma to the neck area was $0.002 \%$ in 1949 [3]. Killing techniques that impact and incarcerate the neck have been used since the early days of humanity; however, high-energy blunt neck trauma, such as those due to an explosion or martial arts competitions, have become more prominent in modern days [4]. Today, the incidence of hyoid bone fractures is $1.15 \%$ in

Manuscript submitted March 1, 2018, accepted March 29, 2018

aDepartment of Emergency Medicine,University of Health Sciences, Haseki Training and Research Hospital, Istanbul, Turkey

${ }^{\mathrm{b}}$ Corresponding Author: Ozgur Sogut, Department of Emergency Medicine, University of Health Sciences, Haseki Research and Training Hospital, Millet Street, Zip Code: 34096, Fatih/Istanbul, Turkey.

Email: ozgur.sogut@sbu.edu.tr

doi: https://doi.org/10.14740/jcs346e martial arts competitions $[2,5]$. The most important protective mechanism is that the hyoid bone situated between the mandible and cervical spine [5,6]. While the mandible preserves the anatomic position of the hyoid, another protective mechanism is fusion of the hyoid bone parts [6, 7]. However, this protection is not entirely effective against hyperextension and blunt traumas.

A hyoid bone fracture caused by blunt trauma is exceedingly rare, except during strangulation and hanging [8]. Therefore, it may go undetected during a physical examination and could cause a life-threatening airway obstruction $[1,5]$. Injuries related to blunt neck trauma may quickly lead to lifethreatening situations and an incorrect or late diagnosis may result in morbidity and mortality [4]. Thus, appropriate measures should be taken to carefully examine patients who have suffered blunt head and neck trauma [9]. To date, few cases of hyoid bone fractures caused by blunt neck trauma have been reported [1, 5, 7-10]. Here, we report a case of a young male with an isolated fracture of the hyoid bone secondary to blunt neck trauma due to a traffic accident.

\section{Case Report}

A 21-year-old male was admitted to our emergency department (ED) following a traffic accident complaining of anterior neck pain. The patient stated that he was sitting in the back seat of an automobile with his seatbelt unfastened at the time of a head-on collision. As a result, he hit his head on the edge of the front seat, which forced his neck back. On presentation to our $\mathrm{ED}$, his blood pressure was $110 / 80 \mathrm{~mm} \mathrm{Hg}$, his pulse rate was 86 beats per min, his respiratory rate was 13 per min, his body temperature was $36.8{ }^{\circ} \mathrm{C}$, and his oxygen saturation was $99 \%$ while breathing room air. Neck movements were unrestricted but the patient was extremely agitated because of conspicuous neck pain. In addition, his right cricoid bone was tender during palpation. No loss of consciousness had occurred, and the Glasgow Coma Scale was 15/15. The patient's pupils were equal and reactive to light, and no midline bony tenderness was detected in the cervical spine upon palpation. Anteroposterior and lateral cervical radiographs obtained on admission showed normal cervical spine, and neck structures. A lateral cervical radiograph demonstrated a slight fracture line and displacement of the hyoid bone (Fig. 1a, b).

A cervical computed tomography $(\mathrm{CT})$ scan was performed 


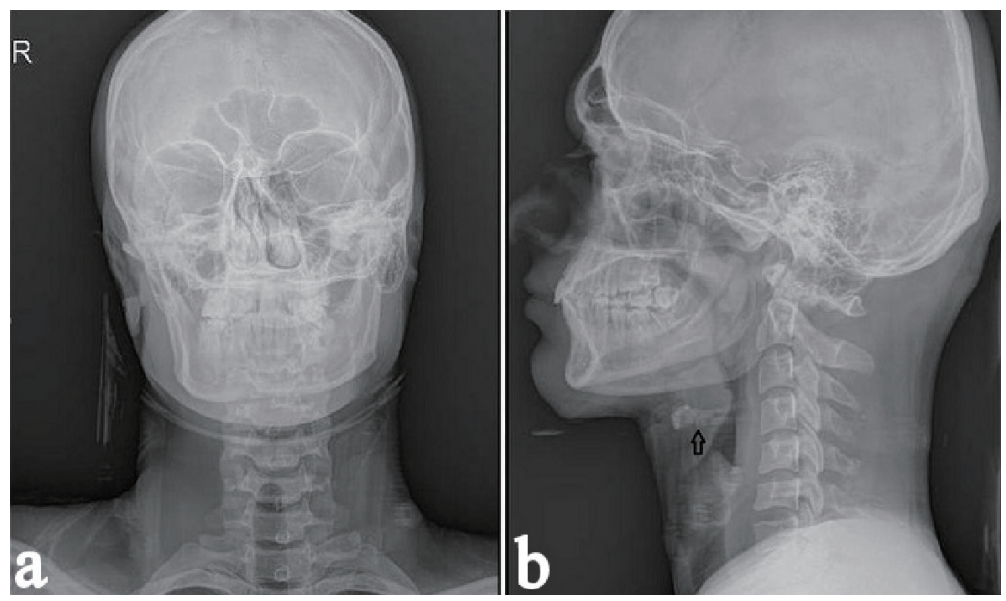

Figure 1. (a) Antero-posterior cervical radiograph obtained on admission showing the normal structures of the neck region. (b) Lateral cervical radiograph obtained on admission showing a subtle fracture line and displacement (arrow).

because of persistent neck pain and it revealed a conspicuous fracture of the right greater horn of the hyoid bone with local swelling of adjacent soft tissue (Fig. 2). Consequently, the patient was referred to the otorhinolaryngology department. Indirect transoral laryngoscopy showed symmetrical motion of the bilateral vocal cords with no edema or lacerations of the airway.

The patient's oral intake was stopped, and he was monitored for an airway obstruction in the ED. In addition, he was treated with oral analgesics and a cold compress was applied to the neck. There was no subcutaneous emphysema or compromised airways during a physical examination. His vital signs remained stable and he was discharged after $24 \mathrm{~h}$, with a recommendation to limit neck movement and consume a liquid or soft diet for a few days. He was told to return to the otorhinolaryngology department for a follow-up exam.

\section{Discussion}

This index case focuses on managing a patient with an uncommon isolated fracture of the right greater horn of the hyoid bone sustained in a traffic accident. The patient had no concomitant injuries. Blunt neck trauma is usually caused by a motor vehicle accident, strangulation, sport injury, or assault [9]. The force of impact is 4 tons in the case of a head-on collision at a speed of $50 \mathrm{~km} / \mathrm{h}$ on an urban road. Of the fatal traumas caused by this force in a case with no fastened seatbelt, $48 \%$ are head and neck injuries, 38\% are chest-abdomen-hip injuries and $8 \%$ are spine and chest wall injuries [11].

In the present case, direct blunt trauma in the form of a head-on collision with his seatbelt unfastened at the time of injury was delivered to the neck region of the body resulting in hyperextension of the cervical spine.

The hyoid bone is a horseshoe-shaped, solitary bone localized at the level of the third and fourth cervical vertebrae in the front of the neck, which has a body and two greater and two lesser horns [5]. It is located below the protruding part of the mandible, just above the thyroid cartilage as a hanging styloid protrusion that supports movement of the tongue in front of the cervical spine. These structures protect the hyoid bone from direct-impact injury $[10,12,13]$. Although it does not directly make a joint, the hyoid is a sensitive bone on which the cervical and tongue muscles are attached, and is very close to vital formations [13]. Hyoid bone fractures mostly occur in cases of hanging, manual strangulation, and drowning [1, 14]. These

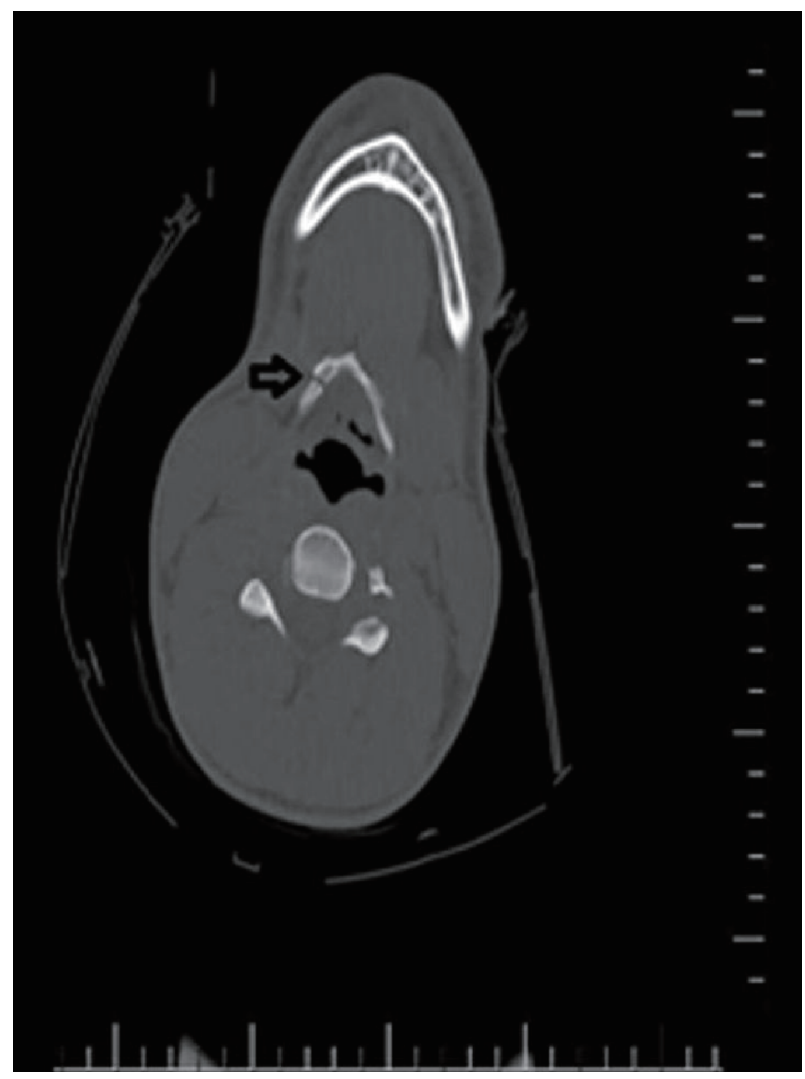

Figure 2. Computed tomography scan of the neck showing a clear fracture in the right greater horn of the hyoid bone with mild adjacent soft tissue swelling (arrow). 
fractures may occasionally be due to direct blunt trauma [15]. The rarity of a hyoid fracture is related to the omnidirectional movement ability of the hyoid bone between the mandible and cervical spine [8]. Protection of the hyoid by the surrounding bones decreases during hyperextension [7]. Even without direct trauma, a hyoid bone fracture can be caused by sudden cervical hyperextension [16].

In the present case, the fracture was due to direct blunt trauma due to hyperextension of the cervical spine. A physical examination, cervical radiographs, a CT scan, and direct laryngoscopy are valuable for diagnosing a hyoid bone fracture [5]. Anteroposterior and lateral cervical radiographs remain the gold standard to diagnose this injury [17]. However, a hyoid bone fracture may not be detected easily on a frontal cervical radiograph and may be relatively subtle on lateral cervical radiographs [12].

Thus, a cervical CT scan should be taken to confirm suspicion of a hyoid bone fracture $[8,12]$. It is also recommended to evaluate the bony and soft tissue structures of the neck, including mandibular fractures, facial fractures, thyroid cartilage fractures, pharyngeal trauma, laryngeal lacerations, cervical spine injuries, vascular trauma, and external carotid artery pseudo-aneurysms $[1,10,12]$.

As in the present case, radiographs may reveal an inconspicuous radiolucent fracture line and displaced hyoid bone fragments, which cast doubt on a hyoid bone fracture. However, in our case, a neck CT scan revealed a clearly displaced right horn of the greater hyoid bone fracture with mild swelling of the adjacent soft tissues. Direct laryngoscopy is recommended to assess edema or lacerations in the upper airway and to evaluate vocal cord motility $[2,5]$. In our case, direct laryngoscopy showed symmetric motility of the vocal cords and no swelling or lacerations in the upper airway.

Symptoms associated with a hyoid bone fracture vary from mild neck pain to acute upper airway obstruction [18]. Swelling of the traumatized area, crepitation, or tenderness with palpation and pain with head movement may occur $[7,12]$. A sharply fractured hyoid bone end can damage the prevertebral muscles and cause hemoptysis, subcutaneous emphysema, and ecchymosis [2]. Although a hyoid bone fracture is rare, it may contribute significantly to the morbidity and mortality of patients with trauma, particularly when concomitant life-threatening injuries are present. A hyoid bone fracture may lead to laryngeal and pharyngeal lacerations and swelling of the anterior cervical tissue and provoke severe upper airway compromise $[8,12]$. The patient in the present case experienced anterior neck pain aggravated by palpation and neck extension. Anterior neck tenderness was noted on palpation. Hemoptysis, subcutaneous emphysema, and ecchymosis related to an upper airway laceration were not detected.

Management of a hyoid bone fracture mostly depends on symptom severity. Conservative treatment is usually adequate for closed or asymptomatic fractures, and the patient rarely requires a surgical intervention $[1,10,12]$. Although most such fractures are treated conservatively and uneventfully, they are potentially life-threatening injuries that occasionally result in severe respiratory compromise [17]. Emergency orotracheal intubation or surgical tracheostomy is warranted to secure the airway in cases of severe airway compromise [12]. Close ob- servation for at least $24 \mathrm{~h}$ is required for asymptomatic fractures to assess airway security [1]. As with conservative therapy, analgesics, fixation with a soft neck rest and a soft or liquid diet may be implemented [5]. An open hyoid bone fracture or symptomatic hyoid bone fracture related to a pharyngeal laceration is an indication for surgical exploration $[1,19]$. In our case, the presenting symptoms did not worsen and did not cause acute airway compromise during observation of the patient in the ED. The patient was observed closely with the head elevated, no oral intake, a soft diet, and administration of analgesics.

\section{Conclusions}

A hyoid bone fracture should be suspected when neck tenderness and pain are detected in the neck region following blunt trauma. A delay in diagnosis may result in life-threatening narrowing of the airway. A CT examination of the neck is the diagnostic modality of choice due to its high accuracy. It is of great importance to closely monitor patients with an isolated closed fracture of the hyoid bone for a prolonged period to assess airway patency. Patients with such a fracture who have no signs of respiratory compromise under close observation can be managed conservatively. Surgical intervention is warranted if symptoms worsen.

\section{References}

1. Erdogan B, Erdogan MO, Colak S, Kibici O, Bozan K, Alper B. An isolated hyoid bone fracture caused by blunt trauma to the neck. J Pak Med Assoc. 2015;65(11):12331234.

2. Chowdhury R, Crocco AG, El-Hakim H. An isolated hyoid fracture secondary to sport injury. A case report and review of literature. Int J Pediatr Otorhinolaryngol. 2005;69(3):411-414.

3. Olmstead EG. Fractures of the hyoid bone; presentation of two cases, with a review of the literature. Arch Otolaryngol. 1949;49(3):266-274.

4. Pollard J, Piercecchi-Marti MD, Thollon L, Bartoli C, Adalian P, Becart-Robert A, Tournel G, et al. Mechanisms of hyoid bone fracture after modelling: evaluation of anthropological criteria defining two relevant models. Forensic Sci Int. 2011;212(1-3):274 e271-275.

5. Porr J, Laframboise M, Kazemi M. Traumatic hyoid bone fracture - a case report and review of the literature. J Can Chiropr Assoc. 2012;56(4):269-274.

6. Gupta R, Clarke DE, Wyer P. Stress fracture of the hyoid bone caused by induced vomiting. Ann Emerg Med. 1995;26(4):518-521.

7. Dalati T. Isolated hyoid bone fracture. Review of an unusual entity. Int J Oral Maxillofac Surg. 2005;34(4):449452.

8. Erdogan MO, Kosargelir M, Yorulmaz R, Meric K, Erdogan B. [Isolated hyoid bone fracture due to blunt trauma: case report]. Ulus Travma Acil Cerrahi Derg. 2013;19(3):282-284.

9. Yauh-Mirng Jehnga, Francis Tzen-TakLee, Yung-Chia 
Pai, Wai-Mau Choi. Hyoid bone fracture caused by blunt neck trauma. Journal of Acute Medicine. 2012;2(3):8384.

10. Keerthi R, Quadri A. Hyoid Bone Fracture: Associated With Head and Neck Trauma-A Rare Case Report. J Maxillofac Oral Surg. 2016;15(Suppl 2):249-252.

11. Strandroth J. Validation of a method to evaluate future impact of road safety interventions, a comparison between fatal passenger car crashes in Sweden 2000 and 2010. Accid Anal Prev. 2015;76:133-140.

12. Cutuk A, Bissell B, Schmidt P, Miller B. Isolated hyoid bone fractures in collegiate football players: a case series and review of the literature. Sports Health. 2012;4(1):5156.

13. Inanir NT, Eren B, Cetin S, Eren F, Gundogmus UN. Anatomical variation of hyoid bone: a case report. Maedica (Buchar). 2014;9(3):272-274.

14. Boussaid M, Mesrati MA, Mahjoub Y, Haj Salem N, Zakhama A, Chadly A, Moussa A, et al. Cervical injuries in drowning cases: a case report and a review of literature. Am J Forensic Med Pathol. 2018;39(1):27-29.

15. Lebreton-Chakour C, Godio-Raboutet $\mathrm{Y}$, Torrents R, Chaumoitre K, Boval C, Bartoli C, Adalian P, et al. Manual strangulation: experimental approach to the genesis of hyoid bone fractures. Forensic Sci Int. 2013;228(1-3):4751.

16. Levine E, Taub PJ. Hyoid bone fractures. Mt Sinai J Med. 2006;73(7):1015-1018.

17. Angoules AG, Boutsikari EC. Traumatic hyoid bone fractures: rare but potentially life threatening injuries. Emergency Med. 2013;3(1):1000e128.

18. Rifai M, Tawab MA. Unidentified hyoid bone fracture and its possible impact on the patient's life: a report of 2 cases. Ann Otolaryngol Rhinol. 2016;3(7):1121.

19. Ramchand T, Choudhry OJ, Shukla PA, Tomovic S, Kuperan AB, Eloy JA. Management of hyoid bone fractures: a systematic review. Otolaryngol Head Neck Surg. 2012;147(2):204-208. 